\title{
Coronavirus disease 2019 in patients with Behcet's disease: a report of 59 cases in Iran
}

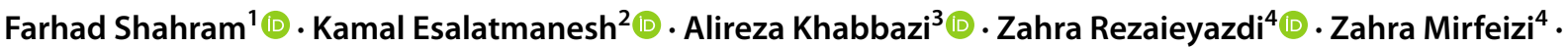 \\ Alireza Sadeghi ${ }^{5}$ (D) Mohsen Soroosh $^{6} \cdot$ Hoda Kavosi $^{1}$ (1) $\cdot$ Majid Alikhani $^{1} \cdot$ Shayan Mostafaei ${ }^{1,7}$ (1)
}

Received: 8 October 2021 / Revised: 10 November 2021 / Accepted: 24 November 2021 / Published online: 29 November 2021

(C) International League of Associations for Rheumatology (ILAR) 2021

\begin{abstract}
Objectives To present the clinical characteristics, disease course, management, and outcomes of COVID-19 infection in patients with Behcet's disease (BD).

Methods In this retrospective cohort study, we retrieved BD patients with definite diagnosis of COVID-19 infection. Demographic data, comorbidities, features related both to BD and COVID-19 infection, treatments, and outcomes were collected. Comparisons between patients with or without hospitalization were performed. All statistical analyzes were performed using SPSS version 25 . We considered $p<0.05$ statistically significant.

Results We identified 61 episodes of COVID-19 infection in 59 BD patients. The prevalence was $0.69 \%$. The median age was 45 years $(I Q R=20)$, and the median disease duration was 162 months $(I Q R=195)$. BD features were similar except for higher rate of arterial involvement and positive pathergy test in infected patients. Thirty-five episodes (62.5\%) happened in non-active patients; $39 \%$ had a comorbid disease. COVID manifestations were the same as the general population. Flu-like symptoms were the most common (85\%), followed by fever (66\%), ageusia/anosmia (56\%), headache (51\%), and pulmonary involvement (48\%). There was no change in BD symptoms in $74 \%$. Fifteen patients (25.4\%) were hospitalized, and one patient (1.7\%) died. Receiving glucocorticoids $(p<0.03)$ and cytotoxic drugs $(p<0.02)$ were associated with an increased rate of hospitalization.

Conclusion The incidence of COVID-19 infection in BD patients was not higher than general population in Iran. They showed milder form of disease with lower morbidity and mortality rate. Most were on immunosuppressive drugs, or had a comorbidity apart from BD. No significant effect on BD course was shown.
\end{abstract}

\section{Key Points}

- The incidence of COVID-19 infection in patients with Behcet's disease is not higher.

- They showed milder form of infection with lower morbidity and mortality rate.

- No significant effect on Behcet's disease course was shown with COVID19 infection.

- BD patients can be managed according to the guidelines used for general population.

Keywords Behcet's disease · COVID-19 · Outcome · Vasculitis

Farhad Shahram

shahramf@tums.ac.ir

1 Behcet's Disease Unit, Rheumatology Research Center, Shariati Hospital, Tehran University of Medical Sciences, Kargar Avenue, 14117-13137 Tehran, Iran

2 Autoimmune Diseases Research Center, Kashan University of Medical Sciences, Kashan, Iran

3 Connective Tissue Diseases Research Center, Tabriz University of Medical Sciences, Tabriz, Iran
4 Rheumatic Diseases Research Center, Ghaem Hospital, Mashhad University of Medical Sciences, Mashhad, Iran

5 Department of Internal Medicine, School of Medicine, Zanjan University of Medical Sciences, Zanjan, Iran

6 AJA University of Medical Sciences, Tehran, Iran

7 Division of Clinical Geriatrics, Department of Neurobiology, Care Sciences and Society, Karolinska Institutet, Stockholm, Sweden 


\section{Introduction}

Since December 2019, a novel infection with severe acute respiratory syndrome coronavirus 2 (SARS-Cov-2) broke out in Wuhan, China, and has transformed into a worldwide challenge. The disease, named coronavirus disease 2019 (COVID-19), was officially declared a pandemic by World Health Organization (WHO) on 11 March 2020 [1].

The risk of COVID-19 and its associated complications is considered to be increased in patients with preexisting medical conditions [2]. Whether patients with autoimmune diseases or vasculitides are at increased risk of COVID-19 is not established yet [3]. In general, immunosuppression and the presence of comorbidities are associated with an increased risk of serious infection in people with rheumatic diseases [4]. However, to date, it has not shown a higher incidence of COVID-19 in these patients [5], even if receiving biologic disease-modifying antirheumatic drug (b-DMARD) therapy [6, 7].

It is still unclear, if there is a modified course of COVID-19 in patients with autoimmune and/or auto-inflammatory diseases [3, 8-10], and whether they are at higher risk for a more severe course with COVID-19, including hospitalization, complications, and death [4]. In addition, a preliminary survey study showed that patients treated with DMARDs and infected with COVID-19 did not develop lifethreatening complications due to their underlying medication [11].

During the pandemic of COVID-19, a proportion of the autoimmune disease patients suspended their medication due to fear of the immunosuppressive effect of medications or lack of availabilities [12], and decreased medical visits because of concerns of the contagious nature of SARSCoV-2 [13]. Disrupted continuity of medical care and medication non-adherence are associated with rheumatologic disease flares and worsened disease activity [14].

Behcet's disease (BD) is a chronic multi-system disease [15], classified as a variable type vasculitis due to affecting vessels of all sizes [16]. Its clinical picture is dominated by recurrent oral and genital ulcers, skin manifestations, and ocular lesions. Other manifestations are related to the involvement of joints, large vessels, the nervous system, and the gastrointestinal tract [15].

Data on the course of COVID-19 in BD patients remain scarce and limited to small case series [17].

In this study, we present a large case series of COVID-19 in patients with $\mathrm{BD}$ and describe their clinical characteristics, disease course, management, and outcomes.

\section{Materials and methods}

\section{Study design}

This was a retrospective cohort study on BD patients visited between March 2020 and March 2021.

\section{Case definition and selection}

All patients had BD classified by either International Criteria for Behcet's Disease (ICBD) or International Study Group (ISG) criteria [18, 19]. Diagnosis of COVID infection was relied on positive reverse transcription polymerase chain reaction (PCR) test (nasopharyngeal swab samples) and/or typical lesions on the lung high resolution computed tomography (HRCT) in compatible clinical setting [20].

\section{Data collection}

Demographics, BD characteristics, comorbidities other than BD, and features related to COVID-19 infection were collected for each patient. BD characteristics included disease duration, clinical manifestations, disease activity, and medications used at the time of infection. COVID-19 features included symptoms and signs, pharmacological treatments, and clinical outcomes (including hospitalization, morbidity, and death). We also assessed whether patients had any exacerbation of BD during infection. Finally, we checked the effect of different data on hospitalization of patients.

\section{Statistical analysis}

Number (\%) was presented for categorical variables. The mean and the standard deviation (SD) or median and the interquartile range $(I Q R)$ were calculated for the continuous normal and non-normal distributed variables respectively. Kolmogorov-Smirnov test was used to check the normality distribution of the continuous variables. Either the independent $t$ test or the Mann-Whitney $U$ test was used to compare the continuous variables, and the chi-square test with Yates correction was used to compare qualitative or categorical variables. Two-tailed $p$ value of less than 0.05 was considered statistically significant. All statistical analyzes were performed using SPSS version 25 (SPSS Inc., Chicago, IL, USA).

\section{Ethical consideration}

The study protocol was approved by the Research Committee of Rheumatology Research Center (RRC) and the Ethics Committee of Tehran University of Medical Sciences. The study was conducted in accordance with the Code of Ethics of the World Medical Association (Helsinki Declaration of 1975/83) for experiments involving humans and Good Clinical Practice guidelines.

\section{Results}

We identified 61 episodes of COVID-19 infection in 59 patients with $\mathrm{BD}$ (two with recurrence of the infection) in a cohort of 8586 patients. The prevalence rate was $0.69 \%$ in 
our BD cohort. These patients were retrieved from 6 rheumatology academic centers in 5 cities in Iran (Tehran: RRC 33/7773 and AJA University 2/50; Mashhad: 9/186; Tabriz: 6/383; Kashan: 6/74; Zanjan: 3/120). The majority have been diagnosed elsewhere, and contacted us during their routine follow-up visits or for consulting about their disease status and medication after infection. Due to national test shortages, testing was not done systematically for all our BD cohort and it was done only for BD patients complaining of any of the symptoms suggestive of COVID infection. The testing rate was $5.5 \%$, with a test positivity rate of $9.0 \%$ in the BD cohort.

All had confirmed BD diagnosis, 56 (95\%) were classified by the ICBD criteria and $45(76.3 \%)$ by the ISG criteria. Diagnosis of COVID infection was relied on positive PCR test in 52 (88\%) cases, and typical lesions on the lung HRCT with compatible clinical setting in 7 other cases (with positive serum anti-COVID antibody in two of them).

\section{Demographics}

The median age was 45 years $(I Q R=20)$, with age range between 20 and 80. Most were in their fourth to sixth decade of life (20-29: 3, 30-39: 18, 40-49: 14, 50-59: 16,>60: 8). Thirty-two (54\%) were female. The BD duration was 0 to 456 months, with a median of 162 $(I Q R=195)$. Nine percent (5/54) had positive familial history for BD.

\section{BD clinical features}

Different manifestations of BD in infected patients (during their disease course before COVID-19 infection) were as follows: oral aphthosis: 57 (97\%); genital ulcers: 30 (51\%); skin involvement: 30 (51\%), pseudofolliculitis/acneiform lesions: 20 (34\%), erythema nodosum: 17 (29\%); ocular lesions: 34 (58\%), uveitis: 31 (53\%), retinal vasculitis: 25 (42\%); constitutional symptoms: 5 (8\%); joint manifestations: $33(56 \%)$; vascular involvement (venous): 10 (17\%); neurological manifestations (central): 4 (7\%); gastro-intestinal involvement: 4 (7\%); epididymitis: $2 / 27$ (7\% of men); positive pathergy test: 33/52 (63\%); and positive HLA-B5/B51: 30/54 (56\%).

\section{Disease activity}

Data regarding disease activity were available for 56 episodes of infection. Twenty-one (37.5\%) cases were seen in patients with active BD according to the physician global assessment (14 with mild and 7 with moderate disease activity). Thirtyfive episodes $(62.5 \%)$ happened in non-active BD patients.

\section{Medications used when infected}

Except 4 patients who were off treatment, the other 55 (93\%) were using one of the following drugs either alone or in combination: corticosteroids: 42 (76\%); immunomodulators: $33(60 \%)$ (colchicine 30, sulfasalazine 4, mycophenolate 1, levamisole 1); cytotoxic drugs: 35 (64\%) (azathioprine 19, methotrexate 15, cyclophosphamide 2); biologic drugs 6 (11\%) (four anti-TNFs and two Interferon alpha).

\section{Comorbidities}

Twenty-three (39\%) had a comorbid disease apart from BD, including diabetes mellitus (10), hypertension (7), obesity (7), cardiac (6), pulmonary (3) renal disease (2), malignancy, brain abscess and smoking (each in one). Thirteen patients (56.5\%) had single comorbid disease, but 6 had two, 3 had three, and 1 had four concomitant comorbid diseases.

\section{COVID-19 symptoms and signs}

They were the same as seen in the general population. Flulike symptoms (fatigue and myalgia) were the most common, seen in 52 cases (85\%), fever in $40(66 \%)$, headache in $31(51 \%)$, loss of taste and anosmia in $34(56 \%)$. Pulmonary involvement was seen in 29 patients (48\%), but pulmonaryrelated symptoms were seen more: cough in 36 (59\%), dyspnea or chest tightness in 27 (44\%) and expectoration in 9 (15\%) patients. Gastrointestinal symptoms (nausea, vomiting and diarrhea) were seen in 25 patients (41\%). Prevalence of other signs was as follows: cold symptoms (sore throat and nasal congestion) in 12 (20\%); shivering in 20 (33\%); vertigo in 4 (7\%); illusion/restlessness/agitation in $3(5 \%)$; weight loss, sweating, menorrhagia, hypotension, and hypertension each in 1 patient. We had no skin involvement.

\section{Effect on BD status}

There was no change in BD symptoms in 45 cases (74\%). Sixteen patients (26\%) presented with a disease flare during COVID infection, but only 3 of them needed treatment intensification. In more than $95 \%$, the medications were either not changed (44 cases, $72 \%$ ) or even decreased or discontinued (14 cases, $23 \%$ ).

\section{Treatment regimen for COVID}

Seven patients $(11.5 \%)$ received only symptomatic treatments. Thirty patients (49\%) received antibiotics (azithromycin: 22 , ceftriaxone: 2 , ciprofloxacin: 2 , doxycycline: 2 co-amoxiclav: 2, penicillin: 1, tazocin: 1). Antiviral drugs were used in 11 patients (18\%) (sofosbuvir/daclatasvir: 5 , remdesivir: 3 , favipiravir: 1 , both remdesivir and favipiravir: 1, lopinavir/ritonavir: 1). Corticosteroids was administered in 13 patients (21\%), hydroxychloroquine in $10(16.4 \%)$, interferon beta in $4(6.5 \%)$, and anti-TNF in one $(1.7 \%)$. We 
did not access to the treatment regimen for 8 episodes of infection.

\section{Outcome}

Fifteen patients $(25.4 \%)$ were hospitalized, and one patient (1.7\%) died due to severe respiratory failure. We had BD flare in 16 patients (27\%); one patient developed pulmonary embolism after deep vein thrombosis; COVID re-infection was seen in two cases.

\section{Comparison}

Comparing hospitalized and non-hospitalized BD patients with COVID-19 infection showed no significant difference for age, gender, disease duration, individual clinical features, or comorbid diseases. However, the rate of active disease was significantly higher in the hospitalized patients $(58 \%$ vs. $32 \%, p=0.02)$. We found that receiving glucocorticoids $(100 \%$ vs. $59 \%, p<0.04)$ and cytotoxic drugs ( $87 \%$ vs. $48 \%$, $p<0.02$ ) were significantly associated with an increased rate of hospitalization, but not biologic therapies and immunomodulators (Tables 1 and 2).

Comparison of clinical presentation of COVID-19 in BD patients by hospitalization status showed no significant differences except for pulmonary involvement which was higher in hospitalized patients (87\% vs. $35 \%, p=0.001)$. Corticosteroid ( $58 \%$ vs. $15 \%, p=0.006)$, anti-viral (58\% vs. $10 \%, p=0.001)$, and anti-inflammatory drugs $(75 \%$ vs. $39 \%$, $p=0.06$ ) were prescribed more in hospitalized patients, as expected. In addition, COVID-19 infection had less significant effect on $\mathrm{BD}$ treatment in higher percentage of nonhospitalized patients ( $80 \%$ vs. $47 \%, p<0.03)$ (Table 3$)$.

\section{Discussion}

The first official report of coronavirus disease in Iran was in February 2020. As of March 21, 2021, according to both Johns Hopkins University and WHO reports Iran (with a population of $84,770,256$ ) was rated as the fifteenth country in the world by the prevalence with the total number of $1,808,422$ confirmed cases $(21,333 /$ million population) and total death of 61,877 (730/million population). The total tests done was $12,221,274(144,169 /$ million population) $[21,22]$.

In comparison with general population of Iran, both the prevalence and death rate of COVID-19 infection were lower in BD patients $(0.69 \%$ versus $2.13 \%$ and $1.7 \%$ versus $3.42 \%$ respectively). Lower number of testing in BD patients $(5.5 \%$ versus $14.4 \%)$ may be one of the attributing causes. Test positivity was also lower in BD patients $(9.0 \%$ versus $14.8 \%)$. This was in line with report of the
Table 1 Demographic features and clinical characteristics of patients with Behcet's disease diagnosed with COVID-19 (comparison by hospitalization status)

\begin{tabular}{lllr}
\hline & Hospitalized & Non-hospitalized & $p^{*}$ \\
\hline Sex (female/male $\dagger)$ & $7 / 8(47 / 53)$ & $25 / 19(57 / 43)$ & 0.70 \\
Age (years) $\ddagger$ & $48.9(10.5)$ & $45.4(12.3)$ & 0.34 \\
Disease duration (month) $\ddagger$ & $169.3(93.9)$ & $185.1(139.1)$ & 0.73 \\
Active disease $\dagger$ & $7(58)$ & $14(32)$ & 0.09 \\
Familial history for BD $\dagger$ & $3 / 15(20)$ & $2 / 39(5)$ & 0.24 \\
BD criteria $\dagger$ & & & \\
ISG $§$ & $13(87)$ & $32(73)$ & 0.45 \\
ICBD\|l & $14(93)$ & $42(95)$ & 0.72 \\
Medications $\dagger$ & & & \\
Disease modifying & $6 / 15(40)$ & $27 / 46(59)$ & 0.33 \\
Cytotoxic drugs & $13 / 15(87)$ & $22 / 46(48)$ & $<\mathbf{0 . 0 2}$ \\
Biologic drugs & $2 / 15(13)$ & $4 / 46(9)$ & 0.98 \\
Corticosteroids & $15 / 15(100)$ & $27 / 46(59)$ & $<\mathbf{0 . 0 4}$ \\
Comorbid diseases $\dagger$ & $8 / 15(53)$ & $15 / 44(34)$ & 0.31 \\
\hline
\end{tabular}

${ }^{*}$ Corrected by Yates method for chi-square test; †number (\%); $\ddagger$ mean (SD); §International Study Group; II International Criteria for Behcet Disease

Table 2 Clinical features of Behcet's disease in patients with COVID-19 infection (comparison by hospitalization status)

\begin{tabular}{llll}
\hline & Hospitalized* & Non-hospitalized* & $p^{\dagger}$ \\
\hline Patients number & 15 & 44 & \\
Oral ulcer & $14(93)$ & $43(98)$ & 0.98 \\
Genital ulcer & $7(47)$ & $23(52)$ & 0.93 \\
Skin lesions & $7(47)$ & $23(52)$ & 0.93 \\
Pseudofolliculitis & $3(20)$ & $17(39)$ & 0.31 \\
Erythema nodosum & $5(33)$ & $12(27)$ & 0.90 \\
Ocular lesions & $10(67)$ & $24(55)$ & 0.60 \\
Uveitis & $9(60)$ & $22(50)$ & 0.71 \\
Retinal vasculitis & $8(53)$ & $17(39)$ & 0.48 \\
Constitutional & $2(13)$ & $3(7)$ & 0.80 \\
Joint manifestations & $10(67)$ & $23(52)$ & 0.50 \\
Vascular involvement & $2(13)$ & $8(18)$ & 0.97 \\
Central nervous system & $2(13)$ & $2(5)$ & 0.56 \\
$\quad$ lesions & & & \\
Gastrointestinal involve- & $1(7)$ & $3(7)$ & 0.56 \\
$\quad$ ment & & & 0.88 \\
Epididymitis (males) & $1 / 8(12)$ & $1 / 19(5)$ & 0.80 \\
Positive pathergy test & $9 / 14(64)$ & $24 / 38(63)$ & 0.65 \\
Positive HLAB5/B51 & $9 / 14(64)$ & $21 / 40(53)$ & \\
\hline
\end{tabular}

*Number (\%); †corrected by Yates method for chi-square test

International Society for Behcet's disease in January 2021, showing lower overall prevalence of COVID-19 infection in BD patients throughout the world. It might be due to BD patients having been especially careful with social shielding. However, underestimation is also possible [17]. 
Table 3 Clinical features of COVID-19 infection in patients with Behcet's disease (comparison by hospitalization status)

\begin{tabular}{lllr}
\hline & Hospitalized* & Non-hospitalized* & $p^{\dagger}$ \\
\hline Episode number & 15 & 46 & \\
Fever & $10(67)$ & $30(65)$ & 0.83 \\
Headache & $5(33)$ & $26(57)$ & 0.20 \\
Flu-like syndrome & $13(87)$ & $39(85)$ & 0.80 \\
Cold symptoms & $3(20)$ & $9(20)$ & 0.73 \\
Shivering & $5(33)$ & $15(33)$ & 0.79 \\
Loss of taste/Anosmia & $8(53)$ & $26(57)$ & 0.93 \\
Cough & $10(67)$ & $26(57)$ & 0.69 \\
Dyspnea/chest tightness & $9(60)$ & $18(39)$ & 0.26 \\
Expectoration & $3(20)$ & $6(13)$ & 0.80 \\
Pulmonary involvement & $13(87)$ & $16(35)$ & $\mathbf{0 . 0 0 1}$ \\
Gastrointestinal symp- & $7(47)$ & $18(39)$ & 0.83 \\
$\quad$ toms & & & 0.70 \\
BD exacerbation & $5(33)$ & $11(24)$ & $<\mathbf{0 . 0 3}$ \\
No effect on BD treat- & $7(47)$ & $37(80)$ & \\
$\quad$ ment & & & $\mathbf{0 . 0 0 1}$ \\
Treatments & & & \\
Anti-viral drugs & $7 / 12(58)$ & $4 / 41(10)$ & 0.06 \\
Anti-inflammatory & $9 / 12(75)$ & $16 / 41(39)$ & \\
$\quad$ drugs & & $6 / 41(15)$ & $\mathbf{0 0 6}$ \\
Corticosteroids & $7 / 12(58)$ & & \\
\hline
\end{tabular}

*Number (\%); †corrected by Yates method for chi-square test

Patients with COVID infection showed similar BD features to the whole cohort patients except for higher rate of joint involvement and positive pathergy test [23]. This was in accordance with a recent report from Italy showing no significant difference in BD manifestations in those with COVID infection [24].

It is not yet known whether patients with rheumatic diseases receiving immunosuppressive therapy are more susceptible to SARS-CoV-2 or not [20, 24]. Meta-regression analysis demonstrated that prior glucocorticoid use was associated with the increased risk of SARS-CoV-2 infection [9, 24]. Most of our patients (93\%) were on immunomodulator or immunosuppressive drugs, and $68 \%$ were on corticosteroids. Dursen et al. reported that none of their 54 BD patients developed COVID-19 infection while 51 were continuing their immunological treatment [25].

In general, the presence of comorbidities is associated with an increased risk of serious infection in people with rheumatic diseases [11]. Only $39 \%$ of our patients had a comorbid disease apart from BD. It is notable that in general population of Iran, around $90 \%$ of patients with COVID infection had at least one comorbid disease.

Disease activity did not appear to be associated with an increased risk of COVID infection in BD patients [4]. In this study, most episodes of infection (62.5\%) happened in non-active BD patients.
The most common symptoms of COVID-19 are fever and cough, which can progress to pneumonia and acute respiratory distress syndrome (ARDS) or multi-organ failure [26]. Additionally, it may predispose to thrombotic disease, both in the venous and arterial circulations [20]. In accordance with previous reports, COVID symptoms and signs in our BD patients were the same as the general population $[2,17$, 27]. Of interest, we had no skin involvement due to COVID infection in BD patients.

As no specific guidelines for the treatment of COVID infection were present for BD patients, the management was done according to the general national guidelines defined by the Ministry of Health. They received symptomatic treatments, antibiotics (mainly azithromycin), antiviral drugs, hydroxychloroquine, corticosteroids, and interferon.

There is a debate on exacerbation of the underlying rheumatic disease during COVID-19 infection [28, 29]. Medication non-adherence and disrupted continuity of medical care are associated with rheumatologic disease flares and worsening disease activity [14]. We had seen no effect on BD symptoms or any need to change the treatment in most cases. We had BD flare only in $26 \%$ of infectious episodes.

There are many reports on the development of autoimmune diseases after SARS-CoV-2 infection, such as cold agglutinin syndrome, autoimmune hemolytic anemia [30, 31], and systemic lupus erythematosus [32]. Interestingly, we had a case with new development of BD after COVID infection. He was a 57-year-old man presented in July 2020 with fever, sore throat, nasal stiffness, cough, fatigue, and myalgia. He developed later mild dyspnea, diarrhea, anosmia, illusion, and restlessness. He had a history of type 2 diabetes and was on glibenclamide. The diagnosis of COVID infection was made clinically without PCR test. Azithromycin was prescribed along with symptomatic treatments at home. All symptoms resolved completely after 4 weeks. Three months later, he developed recurrent oral and genital ulcers, arthralgia, and constitutional symptoms. HLA typing was positive for B51. The diagnosis of BD was reached upon above symptoms and colchicine was started. COVID19 infection may be suggested as a trigger for the appearance of BD symptoms in this case. As SARS-CoV-2 infection can break immune tolerance and trigger autoimmune responses, it is also likely to induce clinical autoimmunity [13].

In contrast to the first case series reported from Turkey suggesting that BD patients have increased risk for severe outcome when infected with COVID-19 [20], the severity of infection was milder in BD patients in Iran. This was in concordance with several reports showing the milder course of COVID infection in inflammatory rheumatologic diseases $[9,29,33,34]$, and supports the theory that immunosuppression may play a protective role during COVID-19 infection [17]. In general, patients with BD had a COVID-19 clinical picture resembling the general population, and the severity 
of COVID-19 infection was milder in them [17, 27, 29]. However, the number of reported BD cases had not been high enough to be able to see the effects of the disease subtypes, activity and medications on the outcome [17].

We found a similar risk of hospitalization, with lower morbidity and mortality rate in BD patients. In line with other reports [4, 9, 34, 35], using glucocorticoids and cytotoxic drugs were associated with an increased rate of hospitalization. We could not identify any other risk factors associated with hospitalization, except for non-significant higher rate of active disease in them, while previous reports showed that older age and the presence of comorbidity in COVID-19 patients with autoimmune diseases are accompanied with a higher risk of hospitalization [9, 34, 35].

As expected, the main reason for hospitalization was pulmonary involvement. The rate of other clinical features of COVID-19 infection was not different in hospitalized patients. They needed more corticosteroid, anti-viral and anti-inflammatory drugs for their infection management. COVID-19 infection had no effect on the BD treatment in $47 \%$ (compared to $80 \%$ in ambulatory patients).

Only one patient in our series of 59 patients died due to severe respiratory failure, and COVID re-infection was seen in two. Very little is known about the outcomes of patients with COVID-19 who have underlying rheumatic disease $[10,20]$. People with rheumatic diseases may be at higher risk for a more severe course with COVID-19, including hospitalization, requiring intensive care/mechanical ventilation, or death $[4,10,34,36]$. When matched for common comorbidities, the population with rheumatic diseases may not have more frequent death [9,34]. COVID-19 outcomes were worse in those receiving glucocorticoids but not in patients on maintenance anti-cytokine therapy. However, there are reports showing corticosteroid treatment reduce mortality $[9,36]$.

To the best of our knowledge, this is the largest collection of COVID-19 infections among patients with BD, with 61 episodes in 59 cases. There had been few small case series reported from Turkey, Spain, and Italy [20, 24, 27]. In most large studies on COVID infection in patients with rheumatic or autoimmune diseases, BD has not been pointed as a distinct disease and has been included under the large group of vasculitides [3, 9, 33, 34].

There are some limitations in this study. First, it was a retrospective study. Second, we were not able to include all cases of COVID-19 infection with BD, as we did not routinely test our whole BD population for COVID-19 infection, so asymptomatic patients or those with milder infection or those who could not reach us due to quarantine and other restrictions may not be represented. This might cause selection bias due to factors such as geographical location and disease severity.

\section{Conclusion}

The incidence of COVID-19 infection among BD patients is not higher than that observed in the general population of Iran. BD patients had a COVID-19 clinical picture similar to the general population, with milder severity of infection. We found a similar risk of hospitalization, with lower morbidity and mortality rate in BD patients. Regarding BD features, there was not any significant difference except for higher rate of joint involvement and positive pathergy test. Most infected patients (92\%) were on immunomodulator or immunosuppressive drugs, and near $40 \%$ had a comorbid disease apart from BD. In most cases, COVID infection had no effect on BD symptoms or treatment. Of note, there was a case with new development of BD, suggesting COVID infection as a possible trigger for disease presentation.

Acknowledgements The authors acknowledge Asal Salehi MD for her significant cooperation on data collection.

Author contribution Farhad Shahram: conceptualization; data curation; formal analysis; investigation; methodology; project administration; resources; supervision; validation; writing original draft; review and editing.

Kamal Esalatmanesh: data curation; investigation; validation; review and editing.

Alireza Khabbazi: data curation; investigation; validation; review and editing.

Zahra Rezaieyazdi: data curation; investigation; validation; review and editing.

Zahra Mirfeizi: data curation; investigation; validation; review and editing.

Alireza Sadeghi: data curation; investigation; validation; review and editing.

Mohsen Soroosh: data curation; investigation; project administration; validation; review and editing.

Hoda Kavosi: data curation; investigation; validation; review and editing.

Majid Alikhani: data curation; investigation; project administration; validation; review and editing.

Shayan Mostafaei: formal analysis; investigation; methodology; validation; review and editing.

\section{Declarations}

Ethical approval The study protocol was approved by the Research Committee of Rheumatology Research Center and the Ethics Committee of Tehran University of Medical Sciences. The study was conducted in accordance with the Code of Ethics of the World Medical Association (Helsinki Declaration of 1975/83) for experiments involving humans and Good Clinical Practice guidelines.

Consent to participate A written informed consent was obtained from each participant according to the Declaration of Helsinki.

Consent for publication All authors approved the final version to be published and agreed to be accountable for all aspects of the work.

Disclosures None. 


\section{References}

1. Pollard C, Morran M, Nestor-Kalinoski A (2020) The COVID-19 pandemic: a global health crisis. Physiol Genomics 52:549-557

2. Eslambolchi A, Aghaghazvini L, Gholamrezanezhad A et al (2021) Coronavirus disease 2019 (COVID-19) in patients with systemic autoimmune diseases or vasculitis: radiologic presentation. J Thromb Thrombolys 51:339-348

3. Favalli E, Ingegnoli F, Cimaz R et al (2021) What is the true incidence of COVID-19 in patients with rheumatic diseases? Ann Rheum Dis. https://doi.org/10.1136/annrheumdis-2020-217615

4. Gianfrancesco M, Hyrich KL, Al-Adely S, Carmona L, Danila MI, Gossec L, Izadi Z, Jacobsohn L, Katz P, Lawson-Tovey S, Mateus EF, Rush S, Schmajuk G, Simard J, Strangfeld A, Trupin L, Wysham KD, Bhana S, Costello W, Grainger R, Hausmann JS, Liew JW, Sirotich E, Sufka P, Wallace ZS, Yazdany J, Machado PM, Robinson PC (2020) COVID-19 Global rheumatology alliance. Characteristics associated with hospitalisation for COVID19 in people with rheumatic disease: data from the COVID19 global rheumatology alliance physician-reported registry. Ann Rheum Dis 79(7):859-866. https://doi.org/10.1136/annrh eumdis-2020-217871

5. Pablos J, Abasolo L, Alvaro-Gracia J et al (2020) Prevalence of hospital PCR-confirmed COVID-19 cases in patients with chronic inflammatory and autoimmune rheumatic diseases. Ann Rheum Dis 79:1170-1173

6. Michelena X, Borrell H, Lopez-Corbeto M et al (2020) Incidence of COVID-19 in a cohort of adult and paediatric patients with rheumatic diseases treated with targeted biologic and synthetic diseasemodifying anti-rheumatic drugs. Semin Arthritis Rheum 50:564-570

7. Figueroa-Parra G, Aguirre-Garcia G, Gamboa-Alonso C et al (2020) Are my patients with rheumatic diseases at higher risk of COVID-19? Ann Rheum Dis 79:839-840

8. Welzel T, Samba S, Klein R et al (2021) COVID-19 in autoinflammatory diseases with immunosuppressive treatment. J Clin Med 10:605

9. Akiyama S, Hamdeh S, Micic D et al (2021) Prevalence and clinical outcomes of COVID-19 in patients with autoimmune diseases: a systematic review and meta-analysis. Ann Rheum Dis 80:384-391

10. D'Silva K, Serling-Boyd N, Wallwork R et al (2020) Clinical characteristics and outcomes of patients with coronavirus disease 2019 (COVID-19) and rheumatic disease: a comparative cohort study from a US 'hot spot.' Ann Rheum Dis 79:1156-1162

11. Conticini E, Bargagli E, Bardelli M et al (2021) COVID-19 pneumonia in a large cohort of patients treated with biological and targeted synthetic antirheumatic drugs. Ann Rheum Dis 80:e14. https://doi.org/10.1136/annrheumdis-2020-217681

12. Khabbazi A, Kavandi H, Paribanaem R et al (2020) Adherence to medication in patients with rheumatic diseases during COVID19 pandemic. Ann Rheum Dis. https://doi.org/10.1136/annrh eumdis-2020-218756

13. Liu Y, Sawalha A, Lu Q (2021) COVID-19 and autoimmune diseases. Curr Opin Rheumatol 33:155-162

14. Hassen L, Almaghlouth I, Hassen I et al (2020) Impact of COVID19 outbreak on rheumatic patients' perceptions and behaviors: a cross-sectional study. Int J Rheum Dis 23:1541-1549

15. Davatchi F, Shahram F, Chams C et al (2005) Behçet's disease. Acta Med. Iran 43:233-242

16. Jennette J, Falk R, Bacon P et al (2013) 2012 revised international Chapel Hill consensusconference nomenclature of vasculitides. Arthritis Rheum 65:1-11

17. Zouboulis C, van Laar J, Schirmer M et al (2021) AdamantiadesBehcet's disease (Behcet's disease) and COVID-19. J Eur Acad Dermatol Venereol. https://doi.org/10.1111/jdv.17325
18. Davatchi F, Assaad-Khalil S, Calamia K et al (2014) The international criteria for Behçet's disease (ICBD): a collaborative study of 27 countries on the sensitivity and specifcity of the new criteria. J Eur Acad Dermatol Venereol 28:338-347

19. International Study Group for Behçet's Disease (1990) Criteria for diagnosis of Behçet's disease. Lancet 335:1078-1080

20. Yurttaş B, Oztas M, Tunc A et al (2020) Characteristics and outcomes of Behçet's syndrome patients with Coronavirus Disease 2019: a case series of 10 patients. Intern Emerg Med. https://doi. org/10.1007/s11739-020-02427-8

21. Johns Hopkins University CSSE (2021) Coronavirus Resource Center. https://coronavirus.jhu.edu/region/iran, Accessed 21 March 2021.

22. WHO COVID-19 Dashboard (2021) https://covid19.who.int/ region/emro/country/ir, Accessed 21 March 2021.

23. Davatchi F, Shahram F, Chams-Davatchi C et al (2019) Behcet's disease in Iran; Analysis of 7641 cases. Mod Rheumatol 29:1023-1030

24. Mattioli I, Bettiol A, Silvestri E, Urban ML, Palermo A, Fagni F, Malandrino D, Del Bianco A, Prisco D, Emmi G (2021) Prevalence and clinical course of SARS-CoV-2 infection in patients with Behçet's syndrome. Clin Exp Rheumatol 39 Suppl 132(5):47-50

25. Dursun R, Temiz S, Ozer I et al (2020) Management of patients with Behcet's disease during the COVID-19 pandemic. Dermatol Ther 33:e14063

26. Gandhi R, Lynch J, Del Rio C (2020) Mild or moderate Covid-19. N Engl J Med 383:1757-1766

27. Espinosa G, Araujo O, Amaro S et al (2020) COVID-19 and Behçet's disease: clinical case series. Ann Rheum Dis. https:// doi.org/10.1136/annrheumdis-2020-217778

28. Ye C, Cai S, Shen G et al (2020) Clinical features of rheumatic patients infected with COVID-19 in Wuhan, China. Ann Rheum Dis 79:1007-1013

29. Brito C, Paiva J, Pimentel F et al (2020) COVID-19 in patients with rheumatological diseases treated with anti-TNF. Ann Rheum Dis. https://doi.org/10.1136/annrheumdis-2020-218171

30. Jensen C, Wilson S, Thombare A et al (2020) Cold agglutinin syndrome as a complication of Covid-19 in two cases. Clin Infect Pract. https://doi.org/10.1016/j.clinpr.2020.100041

31. Patil N, Herc E, Girgis M (2020) Cold agglutinin disease and autoimmune haemolytic anemia with pulmonary embolism as a presentation of COVID-19 infection. Hematol Oncol Stem Cell Ther. https://doi.org/10.1016/j.hemonc.2020.06.005

32. Bonometti R, Sacchi M, Stobbione P et al (2020) The first case of systemic lupus erythematosus (SLE) triggered by COVID-19 infection. Eur Rev Med Pharmacol Sci 24:9695-9697

33. Serling-Boyd N, D'Silva K, Hsu T et al (2020) Coronavirus disease 2019 outcomes among patients with rheumatic diseases 6 months into the pandemic. Ann Rheum Dis. https://doi.org/10. 1136/annrheumdis-2020-219279

34. FAI2R, SFR, SNFMI, SOFREMIP, CRI, IMIDIATE consortium and contributors (2020) Severity of COVID-19 and survival in patients with rheumatic and inflammatory diseases: data from the French RMD COVID-19 cohort of 694 patients. Ann Rheum Dis 80:527-538

35. Haberman R, Axelrad J, Chen A et al (2020) Covid-19 in immunemediated inflammatory diseases - case series from New York. $\mathrm{N}$ Engl J Med 383:85-88

36. Haberman R, Castillo R, Chen A et al (2020) COVID-19 in patients with inflammatory arthritis: a prospective study on the effects of comorbidities and disease-modifying antirheumatic drugs on clinical outcomes. Arthritis Rheumatol 72:1981-1989

Publisher's Note Springer Nature remains neutral with regard to jurisdictional claims in published maps and institutional affiliations. 